\title{
Wie Sie das Portal für sich nutzen können
}

\author{
30 Millionen Versicherte der Krankenkassen AOK und BARMER GEK haben \\ ab sofort die Chance, ihren Ärzten online ein Zeugnis auszustellen. Die Ärzte \\ wiederum erhalten dadurch wertvolle Rückmeldungen ihrer Patienten. \\ Darüber hinaus bietet der persönliche Arztbereich des Portals eine ganze \\ Reihe interessanter Funktionen.
}

- Nützt die Online-Arztsuche ausschließlich den Patienten? Weit gefehlt: Das Portal von AOK, BARMER GEK und dem Projekt Weisse Liste ist auch für Ärzte interessant. Praxisteams erhalten über die Online-Arztsuche eine seriöse und methodisch fundierte Rückmeldung. Auch das Online-Portal selbst bietet in einem eigenen Bereich für Ärzte eine Reihe nützlicher Funktionen. Den Arztbereich finden Sie, indem Sie zunächst Ihren eigenen Eintrag im Ergebnisportal suchen. Gehen Sie dazu auf eine der drei Startseiten des Portals:

\section{WWW.}

AOK-Arztnavigator:

www.aok-arztnavi.de

BARMER GEK Arztnavi:

http://arztnavi.barmer-gek.de

Weisse Liste: www.weisse-liste.de/arzt

Klicken Sie auf „Einen Arzt suchen“ und geben Sie Ihr Fachgebiet und die Postleitzahl oder den Ort Ihrer Praxis ein. Alternativ können Sie auf den Reiter „Suche nach Name“ klicken, um durch Eingabe Ihres Nachnamens zu suchen.

In der Ergebnisliste wird neben Kontaktinformationen die „Weiterempfehlungsbereitschaft" der Patienten dargestellt, sobald mehr als zehn Bewertungen für Sie vorliegen. Wenn Sie nun auf Ihren Namen klicken, gelangen Sie zu Ihrem Detailprofil.

Im oberen Teil werden Adresse, Kontaktdaten, Öffnungszeiten und weitere Informationen zu Ihrer Praxis angezeigt. Außerdem finden Sie eine Karte, die den

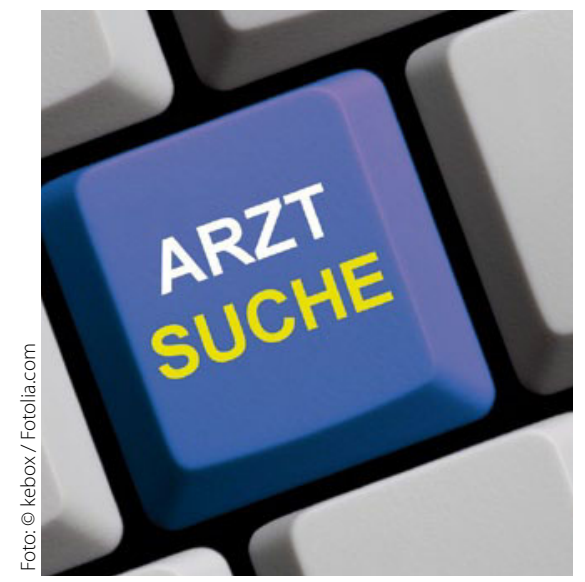

Standort Ihrer Praxis anzeigt. Im unteren Teil der Seite schließen sich die Ergebnisse der Patientenbefragung von AOK, BARMER GEK und Weisse Liste an. Diese werden angezeigt, sobald zehn Bewertungen für Sie vorliegen. Auf der rechten Seite können sich Ihre Patienten ein „Arztprofil“ mit Informationen zu Ihrer Praxis und den Ergebnissen der Patientenbefragung herunterladen.

Unter dem Arztprofil finden Sie den Zugang zu Ihrem persönlichen Portalbereich. Im Folgenden erhalten Sie einen Überblick über die wichtigsten Funktionen:

\section{Korrekturwunsch mitteilen}

Hier finden Sie ein Formular, mit dem Sie Änderungen und Korrekturen mitteilen können, z. B. zu den Adressdaten oder den Öffnungszeiten der Praxis.

Eine Mitteilung ist ohne Authentifizierung möglich und wird redaktionell überprüft. Diese Adress- und Kontakt- daten stammen von der Arzt-Auskunft der Stiftung Gesundheit. Änderungen werden auch dorthin weitergeleitet.

\section{Ergebnisse kommentieren}

Hier können Sie Ihre Ergebnisse für alle Nutzer des Portals sichtbar kommentieren (z. B.: „Unser Wartezimmer ist im Jahr 2010 komplett renoviert worden.“). Für solche Eingaben müssen Sie sich authentifizieren. Dazu können Sie ein Formular herunterladen, um sich per Fax (inklusive Bildausweis) anzumelden. Wenn Sie ein persönliches Nutzerkonto anlegen, müssen Sie nur einmalig Ihre Identität bestätigen. Anschließend können Sie sich jederzeit mit Ihren Benutzerdaten anmelden und alle Funktionen des Arztbereichs nutzen.

\section{Fotos hochladen}

Werten Sie Ihr Profil auf und machen Sie es für die Nutzer des Portals attraktiver, indem Sie es durch Fotos ergänzen! In diesem Portalbereich können Sie bis zu sechs Fotos hochladen.

Vorgesehen sind ein Arztfoto, ein Foto des Praxisteams, ein Bild des Gebäudes sowie mehrere der Praxisräume. Auch für diesen Service müssen Sie sich authentifizieren oder ein persönliches Nutzerkonto anlegen, damit jeder Missbrauch ausgeschlossen ist.

\section{Benachrichtigung abonnieren}

Sie möchten per E-Mail wöchentlich, monatlich oder einmal im Quartal über neue Beurteilungen informiert werden? An dieser Stelle haben Sie die Möglichkeit, eine solche Benachrichtigung zu abonnieren. Auf diese Weise bleiben Sie immer über den aktuellen Stand Ihrer Bewertungen auf dem Laufenden. Selbstverständlich können Sie diesen Dienst jederzeit wieder abbestellen. 